\title{
Discussion to the paper by E. Sherwood Jones
}

LEES. I congratulate Dr Sherwood Jones on a masterly summing up of the part intensive therapy is taking in the present-day medical scene.

We cannot quarrel with the need for intensive therapy, which has been amply demonstrated during this symposium. The lives of critically ill patients can be saved and, equally important, morbidity can be reduced. Evidence is accumulating from prospective studies and on-thespot surveys that the beds needed for intensive therapy fall between $1 \%$ and $2 \%$ of all acute beds. An M.O.H. survey involving 22,363 patients in twenty-four general hospitals (including teaching hospitals) gave this figure and independent observers quote a similar value (St Thomas' Hospital, Whittington, Leeds Royal Infirmary, Whipps Cross). This differs from figures quoted from U.S.A. and the Continent which tend to be much higher. This is presumably because our definition is much more specific; namely, a need for continuous skilled nursing care, frequent attention from doctors and the immediate availability of an extensive range of special equipment.

This symposium has shown that the intensive therapy of a variety of conditions has three main aims: (a) the maintenance of cardio-vascular integrity, (b) the maintenance of respiratory function, and (c) the maintenance of the body fluids. To economize in staff, equipment and facilities one must advocate the concentration of patients in an Intensive Therapy Unit which should be centralized and, whenever possible, contained in one area. The possible exception to this is a regional specialty such as neuro-surgery, cardio-thoracic surgery or cardiology when heavy demands make it worthwhile to set up a separate unit. Such a regional unit should, when possible, be sited adjacent to an existing Intensive Therapy Unit. I would suggest that a district or general Intensive Therapy Unit of less than four beds is too small and available experience indicates that eight beds is an optimum number. These beds are unallocated, essentially short-stay and a bed in a general ward must be available for the patient when the critical phase of his illness has passed. In fact, for practical purposes, it would be better if intensive therapy beds were not listed as 'beds' as such but rather as treatment facilities. Intensive Therapy Units are expensive in capital, equipment, staffing and skills and these factors in themselves necessitate optimal planning and use.

It has been suggested that in order to provide such a concentration of effort, the other phases of progressive patient care in a hospital should be correspondingly diluted and hostel or hotel type accommodation provided for the self-care or minimal-care patient. By definition such patients require no basic nursing care, but may need technical nursing and observation and some medical supervision. In our survey this group amounted to $27 \%$ of all patients in acute wards. Furthermore, assuming the present-day practice of admission and discharge and the use of community medical services some $22 \%$ of patients did not require an in-patient bed. We should ask ourselves why are self-care patients in hospital and what are we doing for them which could not be done as effectively by the community medical and nursing services? My own feeling is that many more experiments are required before we even consider a system of pro- gressive patient care which classifies by nursing needs rather than by disease or sex. Having said this, however, one must agree with the opinion of this symposium that Intensive Therapy Units have proved their worth.

Mrtchell. I agree with Dr Sherwood Jones that it is essential to have Intensive Therapy Units. I am not quite in agreement with him on his definition-I think Intensive Therapy Units and Intensive Care Units need to be defined. There is a difference between the two.

With regard to the nursing aspect in an Intensive Care Unit, with everything a nurse has to do for a patient there is never a moment to spare. It is far better in an Intensive Care Unit to have no spare junior nurses arranging pillows, etc., and getting in the way as a trained Intensive Care nurse must be able to know that even arranging a pillow will increase the patient's respiration and many other things which could interfere with recovery. She must be able to notice these things and know that this will happen. Intensive Therapy is just part of the patient care.

WADE. Dr Lees has spoken about the requirement by Intensive Therapy Units of specially designed or modified buildings. I wonder whether there is not a much more important alteration required in our hospitals if these units are to work efficiently. I refer to the structure of responsibility for patient care. I suspect the day of an individual ward unit under the personal supervision of a single autonomous consultant is approaching its end. In the same way that we now appreciate the benefits that derive if general practitioners work together in a group, so I believe we should accept that consultant physicians or surgeons should combine together to form a Medical of Surgical 'Division'. Part of the work of the 'Division' would be the continuous supervision of the intensive care area.

LEES. There is a great deal to be said for this. The new teaching hospital here is being worked out and planned like that-there will not be individual Consultant or Physician or Surgical beds.

McNicol. Your paper on organization-this paper discussed the organization of many aspects of general management and treatment, feeding, fluid schedules, tracheal intubation and nursing routines but it failed on the critical medical key point of organizing the observation and thinking of the doctor in respect of major treatment decisions. For example, you were unable to say when bronchial lavage should be carried out on the asthmatic case. You made the decision from the foot of the bed and you were unable to describe clearly what features or measurable phenomena influenced you in this decision. If the measurements you were making did not give the answer then these were the wrong measurements and the right measurements should be made and should form the basis of the decisions. One person's view from the foot of the bed is quite different from another's and if this remains the criterion then no progress will be made.

Sherwood Jones. Perhaps this is an error in presentation-of course I did not mean that literally, but meant the charts, etc., at the foot of the bed. I still say that the best assessment for this patient problem (the asthmatic) who is in a critical condition is the chart and records. 

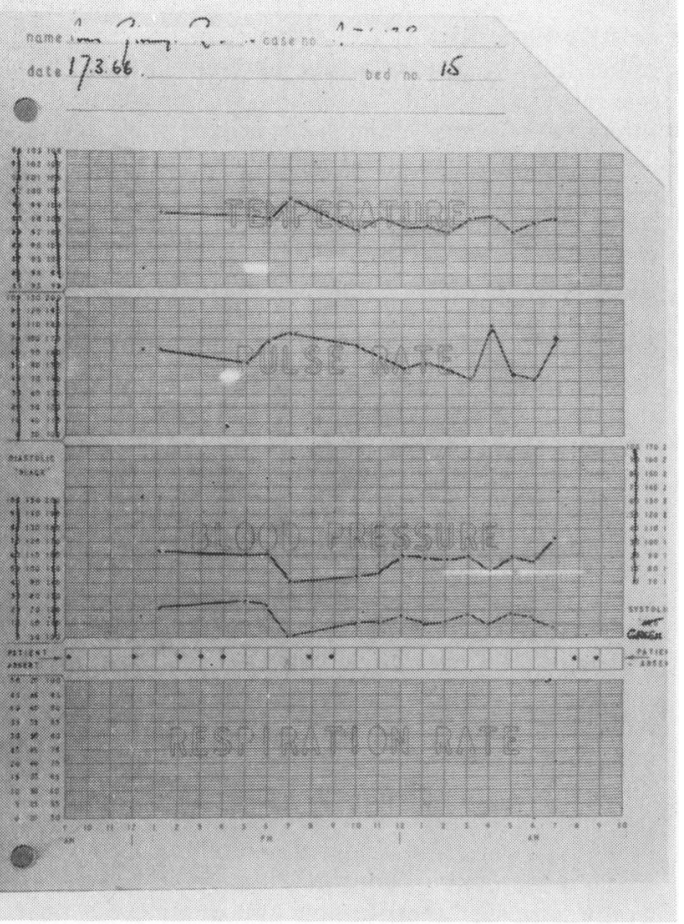

WolfF. There is one subject that has received no $\stackrel{\mathbb{Q}}{\mathscr{Q}}$ mention so far at this conference, and that is the generalc. problem of the presentation of data. This subject happens $\vec{F}$ to be a particular hobby-horse of mine, and I am very grateful to be allowed to ride it for a few minutes.

I am very much afraid that our increasing ability to듬 elicit quantitative data from a patient, be it via patient $\overline{\bar{\omega}}$. monitoring equipment or through an AutoAnalyzer, $\mathbb{\mathbb { D }}$ is far outstripping our ability to assimilate and under- $\bigcirc$ stand the information. Unless steps are taken to minimize this mismatch, much of the information we collect will $\vec{\circ}$ be wasted. Two possibilities are open to us: either we capitulate, admit that the information flow is too large, $\vec{\omega}$ and use a computer to integrate it and to make the de- $\rho$ cisions, or we devise better methods of information: presentation which make it more accessible to the input 3 . equipment connected to the computer between our ears. $\dot{f}$

There is a fundamental difference between the apparent $\omega$ input requirements of an electronic computer and mance which has been largely obscured by the invention of ${ }_{\omega}^{\infty}$ writing. Electronic computers like their input data $\frac{D}{\mathrm{C}}$ presented serially, one piece after the other. This input method would be equivalent to a man reading the table $\stackrel{5}{5}$ in Table A1.

If now all or part of the information content of the $\frac{7}{0}$ table resides in the relationship of one figure to another, either in the same column or in other columns, i.e. trends $\vec{\odot}$ with time or combinations of parameters, this is difficult $\stackrel{\odot}{y}$ to elicit from this form of presentation. The reason for. this is, presumably, that we can only read one figure at

Fig. A1

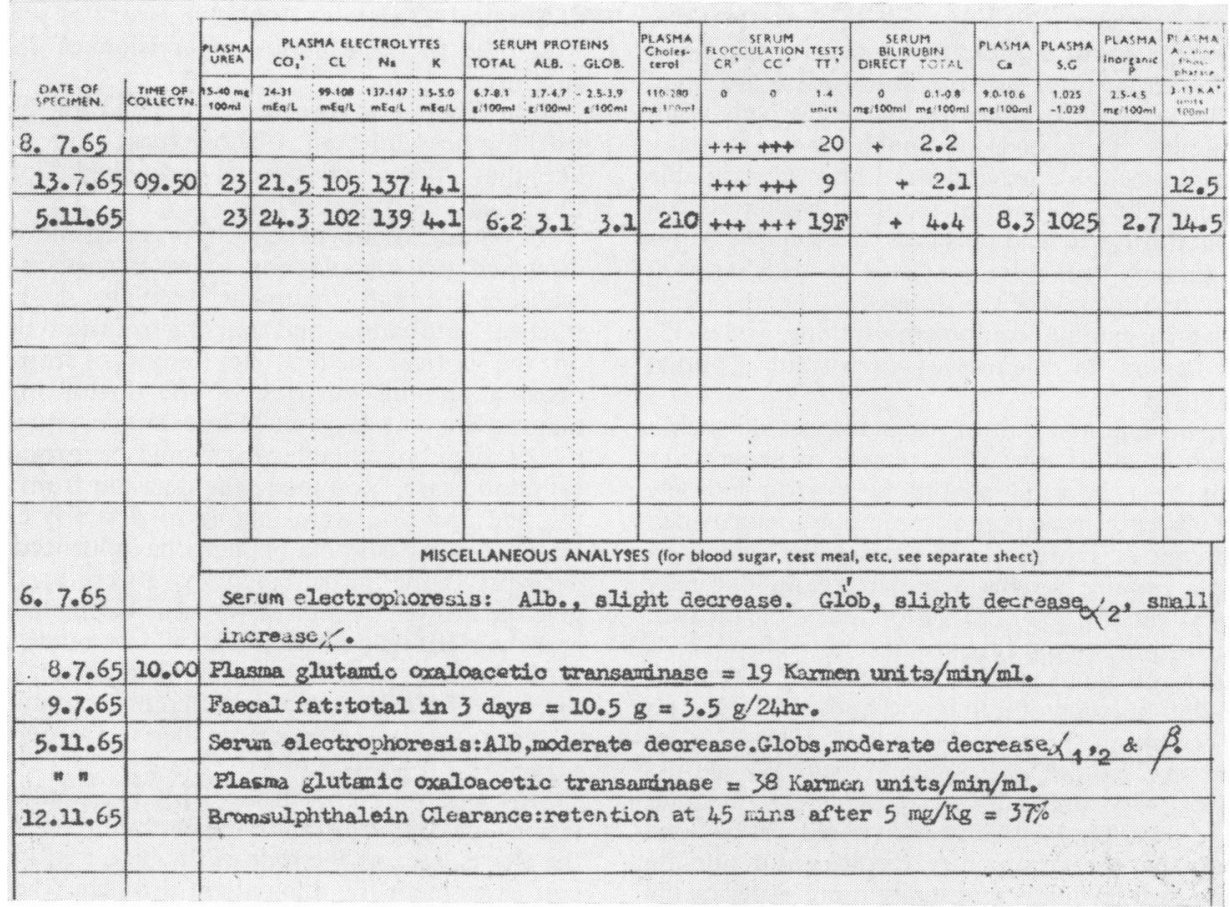


TABLE A1

\begin{tabular}{|c|c|c|c|c|}
\hline $\mathbf{T}$ & $\mathbf{P}$ & $\mathbf{B P}_{\mathbf{s}}$ & $\mathbf{B P}_{\mathrm{d}}$ & $\mathbf{R}$ \\
\hline $37 \cdot 0$ & 75 & 117 & 80 & 15 \\
\hline $36 \cdot 8$ & 75 & 119 & 80 & 16 \\
\hline 36.9 & 76 & 119 & 78 & 17 \\
\hline $37 \cdot 2$ & 76 & 119 & 80 & 17 \\
\hline $37 \cdot 5$ & 77 & 120 & 79 & 17 \\
\hline $37 \cdot 5$ & 80 & 121 & 77 & 18 \\
\hline $37 \cdot 7$ & 83 & 121 & 80 & 20 \\
\hline 38.0 & 84 & 124 & 80 & 20 \\
\hline $38 \cdot 1$ & 91 & 124 & 79 & 21 \\
\hline $38 \cdot 5$ & 93 & 123 & 78 & 21 \\
\hline $38 \cdot 2$ & 95 & 123 & 77 & 22 \\
\hline $38 \cdot 2$ & 95 & 126 & 79 & 22 \\
\hline $38 \cdot 3$ & 90 & 121 & 77 & 20 \\
\hline $38 \cdot 2$ & 90 & 119 & 80 & 19 \\
\hline $38 \cdot 3$ & 85 & 118 & 80 & 20 \\
\hline $38 \cdot 0$ & 83 & 117 & 80 & 20 \\
\hline 38.0 & 80 & 116 & 79 & 19 \\
\hline $37 \cdot 5$ & 77 & 115 & 82 & 19 \\
\hline $37 \cdot 0$ & 77 & 114 & 81 & 19 \\
\hline $37 \cdot 0$ & 78 & 115 & 79 & 21 \\
\hline $37 \cdot 0$ & 74 & 114 & 76 & 19 \\
\hline $36 \cdot 9$ & 73 & 114 & 75 & 19 \\
\hline $37 \cdot 0$ & 72 & 114 & 80 & 18 \\
\hline $36 \cdot 8$ & 72 & 119 & 76 & 18 \\
\hline $37 \cdot 6$ & 72 & 113 & 74 & 19 \\
\hline
\end{tabular}

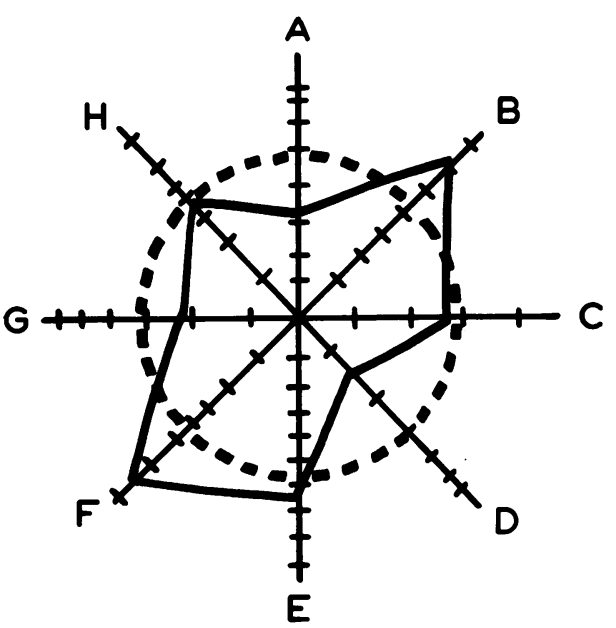

FIG. A3

a time and have no equivalent to the large core-store or memory of the computer in which a representation of the table can be built up. In consequence, to detect trends of relationships we have to make a large number of small experiments using a few figures at a time.

Fig. A1 shows an alternative, and usual, way of presenting data of this type. Trends and relationships are immediately obvious at virtually a single glance, because now we can 'perceive' the whole pattern at once.

Whilst it is unusual to present a time series of physiological measurements in the way shown in Table A1, it is commonplace to report clinical chemistry data on forms, as in Fig. A2. This form, I understand, already represents an improvement in reporting procedure, because the data for investigations on successive days appear on the same piece of paper. However, I feel that it is still very difficult to see relationships between tests
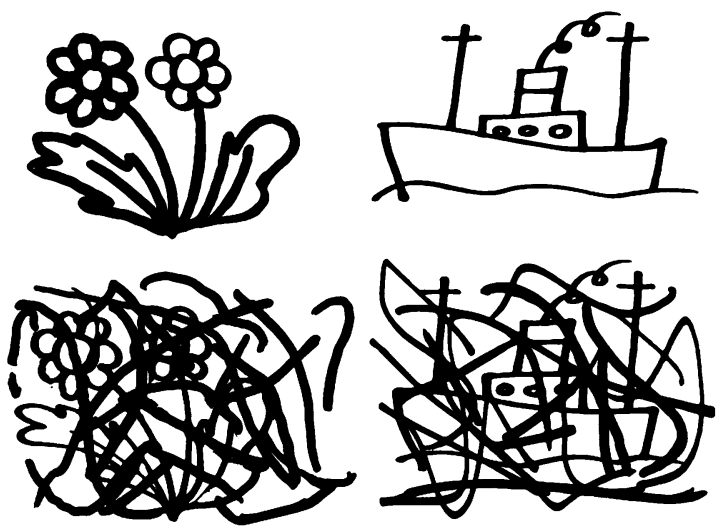

Fig. A4

and that there is room for alternative methods of presentation. For instance, take the scheme illustrated in Fig. A3. Each of the axes $A$ to $H$ is a scale for one parameter. The divisions on the scales are arranged in such a manner that if the points representing normal values are joined a regular octagon approximating to a circle would result (broken line). In practice the actual results are plotted on the axes and joined to form a closed loop (solid line). I believe that by suitable choice of the order of parameters, figures of this type can be used to give an integrated picture of the state of a particular physiological system at a glance. At the same time, the deviation of individual values from the normal is clearly indicated. Displays of this type, for which no originality is claimed, make use of our highly developed shape perception ability, and our shape memory, which could store the loop configuration of many different clinical conditions. If loops for successive days are superimposed (in different colours perhaps) overall progress can also easily be assessed. I do not underrate the effort which would be required to work out an acceptable scheme, even for a limited number of parameters, but in my opinion the contribution this would make to clinical understanding would be inestimable.

Clinical data are essentially noisy, that is not all data elicited are either relevant or even correct. At the present state of the art, decision-making machinery is inferior in these circumstances to ourselves. If one looks at Fig. A4, one has really little difficulty in identifying in the lower part of the diagram the two pictures shown 'clean', namely without noise, in the upper part. This example is visual, but I am sure this ability extends to other forms of input also.

Precisely how this ability to reject noise actually comes about I do not know, but one could postulate that a hypothesis is established on the basis of a small section of the pattern, e.g. this little bit looks rather like a bit of flower, and further evidence is then sought at a location where, if the hypothesis is correct, a high probability exists that it will be found. If the predicted confirmatory evidence is not found, then an alternative hypothesis is tried. I am only elaborating on this example to illustrate further what a powerful data-processing system we possess ourselves, and to make a plea for more appropriate presentation of input material, so as to make better use of human capability. 